OPEN ACCESS

Edited by:

Luc Pieters,

University of Antwerp, Belgium

Reviewed by:

Debomoy K. Lahiri,

Indiana University - Purdue University

Indianapolis, United States

Abdu Adem,

United Arab Emirates University,

United Arab Emirates

${ }^{*}$ Correspondence:

Chihiro Tohda

chihiro@inm.u-toyama.ac.jp

Specialty section:

This article was submitted to

Ethnopharmacology,

a section of the journal

Frontiers in Pharmacology

Received: 24 August 2017

Accepted: 25 October 2017

Published: 14 November 2017

Citation:

Kuboyama T, Hirotsu K, Arai T, Yamasaki $H$ and Tohda C (2017)

Polygalae Radix Extract Prevents

Axonal Degeneration and Memory Deficits in a Transgenic Mouse Model

of Alzheimer's Disease.

Front. Pharmacol. 8:805

doi: 10.3389/fphar.2017.00805

\section{Polygalae Radix Extract Prevents Axonal Degeneration and Memory Deficits in a Transgenic Mouse Model of Alzheimer's Disease}

\author{
Tomoharu Kuboyama ${ }^{1}$, Keisuke Hirotsu², Tetsuya Arai², Hiroo Yamasaki ${ }^{2}$ and \\ Chihiro Tohda ${ }^{\text {* }}$
}

\begin{abstract}
' Division of Neuromedical Science, Institute of Natural Medicine, University of Toyama, Toyama, Japan, ${ }^{2}$ R\&D Center, Kobayashi Pharmaceutical Co., Ltd., Ibaraki, Japan
\end{abstract}

Memory impairments in Alzheimer's disease (AD) occur due to degenerated axons and disrupted neural networks. Since only limited recovery is possible after the destruction of neural networks, preventing axonal degeneration during the early stages of disease progression is necessary to prevent AD. Polygalae Radix (roots of Polygala tenuifolia; PR) is a traditional herbal medicine used for sedation and amnesia. In this study, we aimed to clarify and analyze the preventive effects of PR against memory deficits in a transgenic AD mouse model, 5XFAD. 5XFAD mice demonstrated memory deficits at the age of 5 months. Thus, the water extract of Polygalae Radix (PR extract) was orally administered to 4-month-old 5XFAD mice that did not show signs of memory impairment. After consecutive administrations for 56 days, the PR extract prevented cognitive deficit and axon degeneration associated with the accumulation of amyloid $\beta(A \beta)$ plaques in the perirhinal cortex of the 5XFAD mice. PR extract did not influence the formation of $A \beta$ plaques in the brain of the 5XFAD mice. In cultured neurons, the PR extract prevented axonal growth cone collapse and axonal atrophy induced by $A \beta$. Additionally, it prevented $A \beta$-induced endocytosis at the growth cone of cultured neurons. Our previous study reported that endocytosis inhibition was enough to prevent $A \beta$-induced growth cone collapse, axonal degeneration, and memory impairments. Therefore, the PR extract possibly prevented axonal degeneration and memory impairment by inhibiting endocytosis. PR is the first preventive drug candidate for $A D$ that inhibits endocytosis in neurons.

Keywords: Alzheimer's disease, Polygalae Radix, axon degeneration, amyloid $\beta$, endocytosis, growth cone collapse, 5XFAD mice

\section{INTRODUCTION}

Alzheimer's disease is a progressive, degenerative, and irreversible neurological disorder. Although several clinical drugs are available for $\mathrm{AD}$ patients, these drugs only moderate the progression of $\mathrm{AD}$. None of the $\mathrm{AD}$ treatments have succeeded in recovering cognitive function once the disease has progressed. Thus, since it is too late to treat AD with severe symptoms, a therapy or the

Abbreviations: $\mathrm{A} \beta$, amyloid $\beta$; $\mathrm{AD}$, Alzheimer's disease; ANOVA, analysis of variance; $\mathrm{BSA}$, bovine serum albumin; HPLC, high performance liquid chromatography; NGS, normal goat serum, PBS, phosphate buffered saline; PFA, paraformaldehyde; pNF-H, phosphorylated neurofilament-H; PR, Polygalae Radix; Veh, vehicle; WT, wild-type. 
prevention of $\mathrm{AD}$ at an early stage or before the onset of symptoms is required. Memory deficits are one of the most important core features of $\mathrm{AD}$. Although $\mathrm{AD}$ can develop due to heterogeneous risk factors, such as genome, epigenome, and environments (Killin et al., 2016; Maloney and Lahiri, 2016; Pimenova et al., 2017), the common features of AD include amyloid $\beta(\mathrm{A} \beta)$-induced degeneration of neurites, disruption of neural networks, and memory deficits (Dickson and Vickers, 2001; Hardy and Selkoe, 2002; Perl, 2010; Selkoe and Hardy, 2016). Preventing axonal degeneration could inhibit the progression of memory deficits in $\mathrm{AD}$ (Dickson and Vickers, 2001; Hardy and Selkoe, 2002; Simmons et al., 2014). Additionally, axonal regeneration is related to recovery from cognitive dysfunction in murine models of $\mathrm{AD}$ (Tohda, 2016).

Polygalae Radix (roots of Polygala tenuifolia; PR) is a traditional herbal medicine, which has been clinically used for memory loss in East Asia (Wu et al., 2011; May et al., 2013). There are many reports about the anti-AD properties of PR. For example, PR extracts and their constituents were reported to have a protective effect in cultured neurons from $\mathrm{A} \beta$-induced neurotoxicity ( $\mathrm{Xu}$ et al., 2011); they enhanced axon elongation when cultured neurons were already undergoing $A \beta$-induced axon atrophy (Naito and Tohda, 2006), inhibited $\mathrm{A} \beta$ secretion in cultured cells (Jia et al., 2004; Lv et al., 2009), and recovered memory in intrahippocampally $A \beta$-injected mice (Xu et al., 2011; Liu et al., 2015). Additionally, BT-11, an extract of PR, was reported to recover memory in stress- or scopolamine-induced amnestic rats (Park et al., 2002; Shin et al., 2009), and enhance memory in healthy humans (Lee et al., 2009). Taking these reports into consideration, PR may also have preventive effects against AD. At present, however, there are no studies that have demonstrated that $\mathrm{PR}$ prevents the development of $\mathrm{AD}$ in humans or animal models.

In $\mathrm{AD}$, the increases in the level of $\mathrm{A} \beta$ in the brain precede memory deficits (Jack et al., 2010). A similar phenomenon is also observed in a transgenic mouse model of AD, 5XFAD mice, which expresses mutant human amyloid precursor protein (the Swedish mutations: K670N and M671L; the Florida mutation: I716V; and the London mutation: V717I) and PS1 (M146L; L286V) transgenes, under the neuronspecific mouse Thy-1 promoter (Oakley et al., 2006). A $\beta$ plaques accumulate in the brains of 5XFAD transgenic mice by the age of 2 months, and memory impairments by 4-5 months (Oakley et al., 2006) and axon degeneration by 4-7 months (Tohda et al., 2011, 2012; Yang et al., 2017). This corroborated with the trend seen in patients with AD (Benes et al., 1991; Masliah et al., 1993). In the current study, PR water extract was consecutively administered before the onset of memory impairments in the 5XFAD mice, and its preventive effects on axonal degeneration and memory impairment were investigated. We focused on endocytosis as its inhibition is sufficient to prevent $A \beta$-induced axonal degeneration and memory deficits, and cause these preventive effects (Kuboyama et al., 2015).

\section{MATERIALS AND METHODS}

\section{Polygalae Radix Extract}

The dried powder of the water extract of Polygalae Radix (PR extract; Management No. Q14504) was obtained from Kobayashi Pharmaceutical Co., Ltd. (Ibaraki, Japan). To check the quality of the PR extract, the concentrations of two compounds, i.e., tenuifolin and 3',6-di-O-sinapoyl sucrose ester, were measured using high performance liquid chromatography (HPLC) analysis according to Hong Kong Chinese Materia Medica Standards ${ }^{1}$. These two compounds are index components, which are used to check the quality of PR, according to the Hong Kong Chinese Materia Medica Standards and China Pharmacopoeia (Chinese Pharmacopoeia Commission, 2015). For the quantification of tenuifolin, the PR extract ( $0.15 \mathrm{~g})$ was dissolved in $10 \%$ sodium hydroxide (30 mL; Cat. No. 198-13765; Wako, Osaka, Japan), and the solution was heated and refluxed at $110^{\circ} \mathrm{C}$ for $90 \mathrm{~min}$. The solution was adjusted to $\mathrm{pH} 4-5$ by adding hydrochloric acid (Cat. No. 080-01066; Wako). Water was added to bring the solution up to $100 \mathrm{~mL}$, and $30 \mathrm{~mL}$ of the solution was extracted with 1-butanol (50 mL; Cat. No. 026-03326; Wako) twice. The 1-butanol solution was dried, and then resolubilized with methanol (10 mL; Cat. No. 132-06471; Wako). A total of $10 \mu \mathrm{L}$ of the methanol solution was applied (Shimadzu, Kyoto, Japan) to an HPLC system, on a YMC-Pack ODS-AM AM-312 (6.0 mm i.d. $\times 150 \mathrm{~mm}$, YMC, Kyoto, Japan) column held at $30^{\circ} \mathrm{C}$ with a flow rate of $1 \mathrm{~mL} \mathrm{~min}^{-1}$, and was detected using a photodiode array detector (SPD-M20A, Shimadzu). In the mobile phase, $0.05 \%$ phosphoric acid (v/v) (Cat. No. 167-02166; Wako) and acetonitrile (Cat. No. 015-08633; Wako) (33:17) were used. To quantify 3 ',6-di-O-sinapoyl sucrose ester, the PR extract $(0.3 \mathrm{~g})$ was dissolved in $50 \%$ methanol $(50 \mathrm{~mL})$. A total of $10 \mu \mathrm{L}$ of the $50 \%$ methanol solution was applied to the HPLC system on a Cosmosil 5C18-AR-II (4.6 mm i.d. $\times 150 \mathrm{~mm}$, nacalai tesque, Kyoto, Japan) column held at $30^{\circ} \mathrm{C}$ with a flow rate of

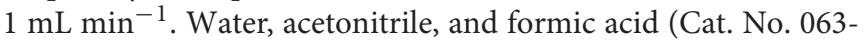
5895; Wako) (860:160:1) were used in the mobile phase. Standard curves were produced to measure concentrations of tenuifolin and 3',6-di-O-sinapoyl sucrose ester in the PR extract. These two compounds were purchased from TOKIWA phytochemical Co., Ltd. (Sakura, Japan) (tenuifolin, Cat. No. P2515; 3',6-di-Osinapoyl sucrose ester, Cat. No. P2939). HPLC profiles of the PR extract are shown in Supplementary Figure S1. Concentrations of tenuifolin and 3',6-di-O-sinapoyl sucrose ester in the PR extract were 2.89 and $0.86 \%$, respectively.

\section{Animal Experiments}

All animal experiments were conducted in accordance with the Guidelines for the Care and Use of Laboratory Animals at the Sugitani Campus of the University of Toyama. The Committee for Animal Care and Use at the Sugitani Campus of the University of Toyama approved all protocols. The approval number for the animal experiments is A2014INM-1 and A2017INM-1, and the confirmation number for the recombinant gene experiments is G2013INM-1.

\footnotetext{
${ }^{1}$ http://www.cmd.gov.hk/html/eng/hkcmms/cmmlist.html
} 
A novel object recognition test was performed using $\mathrm{AD}$ model 5XFAD mice or wild-type (WT) mice (4-month-old, male and female mice; Jackson laboratory, Bar Harbor, ME, United States) as described previously (Kuboyama et al., 2015). Briefly, each mouse was trained to habituate to two identical objects in an open box for $10 \mathrm{~min}$, and were then returned to their home cage. The test session was the performed for $10 \mathrm{~min}$, $22 \mathrm{~h}$ later. In the test session, one of the objects was replaced with a novel object in the same place. The number of times the mouse encountered each object was counted. Preference indices were calculated.

After the test session finished, the PR extract (at a dose of 12 or $60 \mathrm{mg} \mathrm{kg}^{-1} \mathrm{day}^{-1}$ ) or a vehicle solution (sterilized saline) was orally administered, daily, to the mice for 56 days. Saline is an innocuous medium, and treatment with saline is akin to an invasive-free treatment. The day after the last administration, a second trial of the novel object recognition test was performed using different new objects.

\section{Immunohistochemistry}

After the second trial of the novel object recognition test finished, the mice were euthanized and perfusion-fixed with $4 \%$ paraformaldehyde (PFA; Cat. No. 162-16065; Wako) dissolved in phosphate buffered saline (PBS). Brains were cryoprotected in $30 \%$ sucrose and frozen on dry ice. Frozen brains were coronally sectioned at $14 \mu \mathrm{m}$ and further fixed with $4 \%$ PFA at $4^{\circ} \mathrm{C}$ overnight. The sections were then washed with PBS and incubated with blocking solution, i.e., 5\% normal goat serum (NGS; Cat. No. 143-06561; Wako) in a solution of $0.3 \%$ Triton X-100 (Cat. No. 168-11805; Wako) and PBS for $1 \mathrm{~h}$ at room temperature. Then, sections were incubated in a solution containing anti-phosphorylated neurofilament- $\mathrm{H}$ (pNF-H) monoclonal antibody (an axonal marker; dilution 1:500; clone SMI-35; Covance, Dedham, MA, United States), anti-A $\beta$ polyclonal antibody (dilution 1:1000; Cat. No. AB5076; Millipore, Billerica, MA, United States), and 1\% BSA (Cat. No. 010-25783; Wako) in a solution of $0.3 \%$ Triton X-100 and PBS at $4^{\circ} \mathrm{C}$ overnight. Next, the sections were incubated in a solution containing Alexa Fluor 594-conjugated goat anti-mouse IgG (dilution 1:400; Cat. No. A-11005; Thermo Fisher Scientific, Waltham, MA, United States), Alexa Fluor 488-conjugated goat anti-rabbit IgG (dilution 1:400; Cat. No. A-11008; Thermo Fisher Scientific), and $1 \%$ bovine serum albumin (BSA) in a solution of $0.3 \%$ Triton X-100 and PBS at room temperature for $2 \mathrm{~h}$. A series of staining procedures was performed using the Shandon Sequenza slide rack (Thermo Fisher Scientific). Fluorescence images $(325 \mu \mathrm{m} \times 426 \mu \mathrm{m})$ were captured using a $20 \times$ NA 0.8 dry objective lens (Plan-Apochromat, Carl Zeiss, Oberkochen, Germany) and a charge-coupled device camera (AxioCam MRm, binning set at $1 \times 1$, Carl Zeiss) on an inverted microscope (AxioObserver Z1, Carl Zeiss). A total of six images were captured for each brain region in each mouse. The density of $A \beta$ plaques in each brain region and densities of degenerated axons merged on the $A \beta$ plaque were quantified using ImageJ software (National Institutes of Health, Bethesda, MD, United States) as described previously (Tohda et al., 2012).

\section{Collapse Assay}

Cerebral cortical neurons (embryonic day 14; ddY mice; SLC, Hamamatsu, Japan) were cultured in 8-well chamber slides (Falcon, Franklin Lakes, NJ, United States) as described previously (Kuboyama et al., 2015). Three days after the culture started, the neurons were treated with either the PR extract (10 and $100 \mu \mathrm{g} \mathrm{mL}^{-1}$ ) or a vehicle solution (distilled water) for 30 min. Then, they were treated with either full length $A \beta 1-42$ ( $1 \mu \mathrm{M}$; Sigma, St. Louis, MO, United States) or active partial fragment $A \beta 25-35$ (10 $\mu \mathrm{M}$; Sigma) for $1 \mathrm{~h}$, and fixed with $4 \%$ PFA and $4 \%$ sucrose in PBS at $37^{\circ} \mathrm{C}$ for $1 \mathrm{~h}$. A $\beta 1-42$ and A $325-35$ were aggregated before the treatment as described previously (Kuboyama et al., 2015). The most effective doses of $A \beta 1-42$ and $A \beta 25-35$ were selected based on our previous study (Kuboyama et al., 2015). The entire area in each chamber $(7.8 \mathrm{~mm} \times 9 \mathrm{~mm})$ was automatically captured with a $20 \times$ dry objective lens (PlanApo $\lambda$, Keyence, Osaka, Japan) on an inverted microscope (BZ-710, Keyence). Collapse scores were quantified as described previously (Kuboyama et al., 2015). Briefly, the longest neurite in each neuron was judged as an axon, and growth cones (axonal endings) lacking lamellipodia or possessing fewer than three filopodia were judged as collapsed growth cones (Dotti et al., 1988; Jurney et al., 2002). The collapsed growth cones were scored 1 point, while healthy growth cones were scored 0 point. An average of the scores in each treatment group was shown as a collapse score.

\section{Axonal Growth Assay}

After cortical neurons were cultured for 3 days, A $\beta 25-35$ $(10 \mu \mathrm{M})$ was simultaneously treated with PR extract (at doses of $0.1,1,10$, and $100 \mu \mathrm{g} \mathrm{mL}-1$ ) for 4 days. After that, neurons were fixed with $4 \%$ PFA in PBS and blocked with $5 \%$ BSA in a solution of $0.3 \%$ Triton X-100 and PBS for $1 \mathrm{~h}$ at room temperature. The neurons were then incubated in a solution containing anti-pNF-H monoclonal antibody (dilution 1:500), anti-microtubule associated protein 2 polyclonal antibody (a neuronal marker; dilution 1:1000; Cat. No. ab32454; Abcam, Cambridge, United Kingdom), and 5\% BSA in a solution of $0.3 \%$ Triton $\mathrm{X}-100$ and PBS at $4^{\circ} \mathrm{C}$ overnight. Next, the neurons were incubated in a solution containing Alexa Fluor 594-conjugated goat anti-mouse IgG (dilution 1:400), Alexa Fluor 488-conjugated goat anti-rabbit IgG (dilution 1:400), DAPI ( $1 \mu \mathrm{g} \mathrm{mL}^{-1}$, Sigma), and $3 \% \mathrm{BSA}$ and $1 \%$ NGS in a solution of $0.3 \%$ Triton X-100 and PBS for $2 \mathrm{~h}$ at room temperature. Fluorescence images $(650 \mu \mathrm{m} \times 852 \mu \mathrm{m})$ were captured using a $10 \times$ NA 0.4 dry objective lens on an inverted microscope (AxioObserver Z1). The lengths of axons per neuron were measured using MetaMorph 7.8 software (Molecular Devices, Sunnyvale, CA, United States).

\section{Endocytosis Experiments}

Three days after the cortical neurons were cultured, they were treated with either the PR extract $\left(100 \mu \mathrm{g} \mathrm{ml}^{-1}\right)$ or a vehicle solution (distilled water) for $30 \mathrm{~min}$. After that, FM1-43FX (20 $\mathrm{\mu M}$; Thermo Fisher Scientific) was added to the culture medium, and A $\beta 1-42(1 \mu \mathrm{M})$ or vehicle solution (distilled water) 
was added $1 \mathrm{~min}$ later. The neurons were then washed twice with warmed culture medium 20 min later and fixed with 4\% PFA and $4 \%$ sucrose in $\mathrm{PBS}$ for $1 \mathrm{~h}$ at $37^{\circ} \mathrm{C}$. Images of the growth cones were captured using a $63 \times$ NA 1.40 oil imersion objective lens on an inverted microscope (AxioObserver Z1). A total of 50 images were captured for each treatment group. The density of the FM143FX-positive area in the normal growth cones were quantified using ImageJ software as described previously (Kuboyama et al., 2015).

\section{Statistics}

Statistical comparisons were performed using a paired (twotailed) $t$-test, one-way ANOVA followed by a post hoc Bonferroni's multiple comparison test, Kruskal-Wallis test followed by a post hoc Dunn's multiple comparison test, or two-way repeated measures ANOVA. Statistical analyses were performed using Prism 5.04 software (GraphPad software, San Diego, CA, United States). Statistical significance was set at $P<0.05$. The effect sizes $(d)$ were calculated using the $G^{*}$ power 3 statistical software (Faul et al., 2007).

\section{RESULTS}

\section{PR Extract Prevents Memory Impairment in 5XFAD Mice}

5XFAD mice, which have previously shown various AD-like phenotypes, such as $\mathrm{A} \beta$ deposition, phosphorylated tau deposition, neuronal loss, axonal degeneration, and memory deficits (Oakley et al., 2006; Urano and Tohda, 2010; Joyashiki et al., 2011; Tohda et al., 2011, 2012) were used to model AD in the current study. PR was administered before the onset of memory deficits to investigate its preventive effects against AD. The age at which 5 XFAD mice showed signs of memory impairments was determined using 4-month-old 5XFAD or WT mice that underwent a novel object recognition test (Figure 1). In the training session, both groups equally explored each object; the preference indices were approximately 50\%. In the test session, WT and 5XFAD mice significantly increased the time spent exploring the replaced novel object $(P=0.0012, d=3.71$, and $P<0.0001, d=2.80$, respectively). Mice normally prefer a novel object. Therefore, Figure 1 demonstrates that 5XFAD mice and WT mice remembered the familiar object and preferred the novel object. Since cognitive function in the 5XFAD and WT mice seemed to be normal at this age, it was deemed that the cognitive function in the 5XFAD mice was likely to be normal until the age of 4 months. The onset of memory deficits occurred at 5 months of age (Supplementary Figure S2A).

The PR extract or vehicle solution was administered daily to the 4-month-old WT or 5XFAD mice for 56 days. At the age of 6 months, a second trial of the novel object recognition test was performed (Figure 2). There was a significant increase in the preference index in the vehicle-administered WT mice during the test session $(P=0.011, d=2.00)$, whereas there was no increase in the vehicle-administered 5XFAD mice $(P=0.26$, $d=0.58$ ). This finding suggested that memory deficits occurred in 6-month-old 5XFAD mice. In contrast, the administration of a high dose of the PR extract $\left(60 \mathrm{mg} \mathrm{kg}^{-1}\right.$ day $\left.^{-1}\right)$ significantly increased the preference index in the test session for the 5XFAD mice $(P=0.0016, d=3.43)$. The administration of a low dose $\left(12 \mathrm{mg} \mathrm{kg}^{-1} \mathrm{day}^{-1}\right)$ of the PR extract showed a tendency to increase the preference index in the test session $(P=0.080, d=1.31)$. These results demonstrated that the PR extract prevented impairment of object recognition memory in the 5XFAD mice. The administration of PR extract did not influence body weight or exploratory behaviors of the mice (Supplementary Figure S3).

\section{PR Prevents Axonal Degeneration in the Brain of 5XFAD Mice}

After behavioral experiments, brain tissue was extracted from mice and the expression of pNF-H (an axonal marker) and $\mathrm{A} \beta$ in the perirhinal and medial prefrontal cortices of 5XFAD mice were evaluated using immunohistochemistry. A $\beta$ plaques were observed in both regions and bulb-like degenerated axons overlapped the plaques (Figure 3). This was observed in regions related to memory functions (Barker and Warburton, 2011; Vilberg and Davachi, 2013; Tanimizu et al., 2017). We previously confirmed that $\mathrm{A} \beta$ plaques and degenerated axons were observed in these regions of 5XFAD mice (Tohda et al., 2011, 2012; Yang et al., 2017), which corroborated with findings from patients with AD (Benes et al., 1991; Masliah et al., 1993). The administration of PR extract (at doses of 12 or $60 \mathrm{mg}$ $\mathrm{kg}^{-1}$ day $^{-1}$ ) significantly decreased the density of degenerated axons associated with the plaques in the perirhinal cortex (Figures 3A,B). Although PR extract administration (at doses of 12 and $60 \mathrm{mg} \mathrm{kg}^{-1} \mathrm{day}^{-1}$ ) also decreased the density of degenerated axons in the medial prefrontal cortex, this change was not significant (Figure 3C). The density of $A \beta$ deposits in the perirhinal and prefrontal cortices remained unchanged following the administration of PR extract (at doses of 12 and $60 \mathrm{mg} \mathrm{kg}^{-1} \mathrm{day}^{-1}$ ) (Figures 3D,E). Therefore, in 5XFAD mice, the PR extract partially prevented axonal degeneration and cognitive impairment, without affecting $A \beta$ deposition in the brain.

\section{PR Inhibits A $\beta$-Induced Axonal Collapse and Endocytosis}

Degeneration of axonal endings (axonal collapse) is an early event induced by $A \beta$ in cultured neurons (Kuboyama et al., 2015). Both $A \beta 1-42$ and A $\beta 25-35$ induced axonal collapse in cultured neurons (Figure 4) as previously demonstrated (Kuboyama et al., 2015). A $\beta 25-35$ is an active partial fragment of $A \beta$, which is commonly used as an alternative to full length $A \beta 1-42$ and shows similar pharmacological effects to $\mathrm{A} \beta 1-42$, such as neurotoxicity, axonal atrophy, and axonal collapse (Yankner et al., 1990; Kuboyama et al., 2005, 2015). Pre-treatment with the PR extract (at 10 and $100 \mathrm{\mu g}^{-1} \mathrm{ml}^{-1}$ ) prevented $\mathrm{A} \beta 1-42$ - and $\mathrm{A} \beta 25-35$ induced axonal collapses. A $\beta 25-35$ induced axonal atrophy in cultured neurons (Figure 5) as previously shown (Kuboyama et al., 2005). A $325-35$-induced axonal atrophy was also prevented by simultaneous treatment with the PR extract at doses of 10 and $100 \mu \mathrm{g}^{-1} \mathrm{ml}^{-1}$ (Figure 5). 


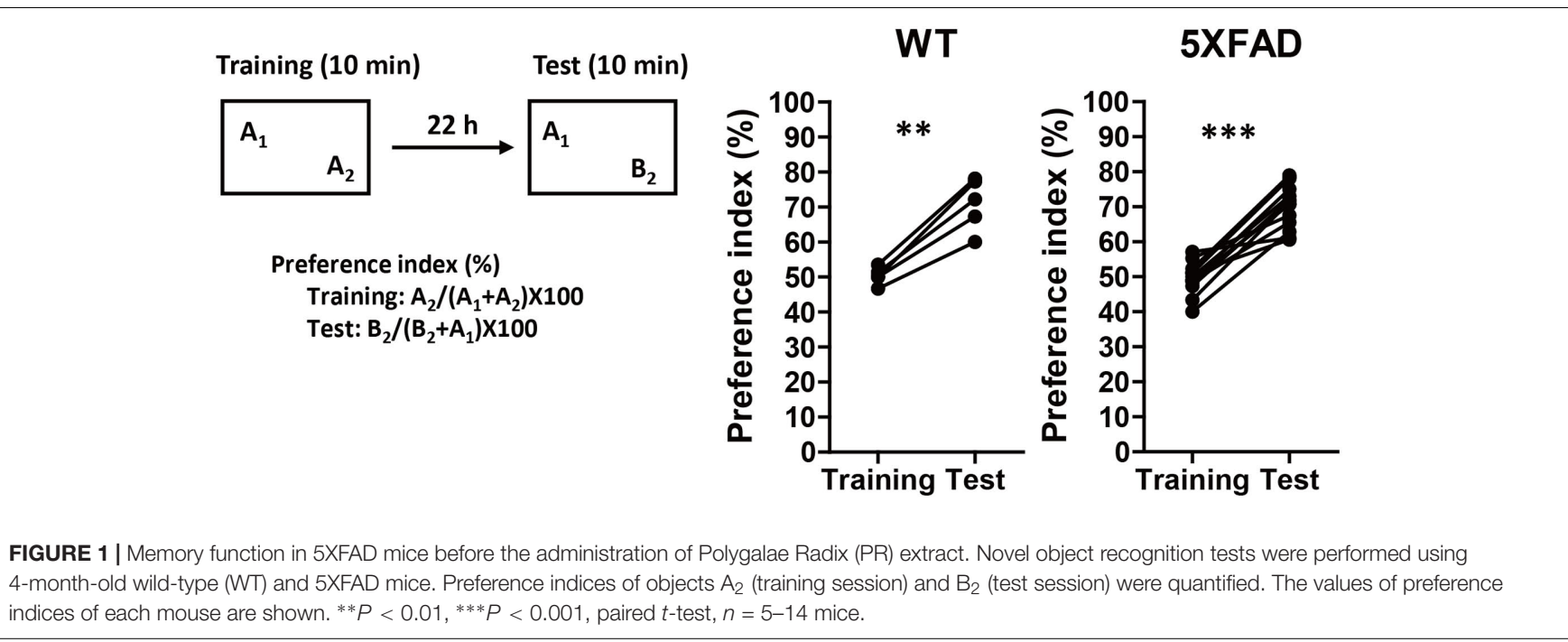

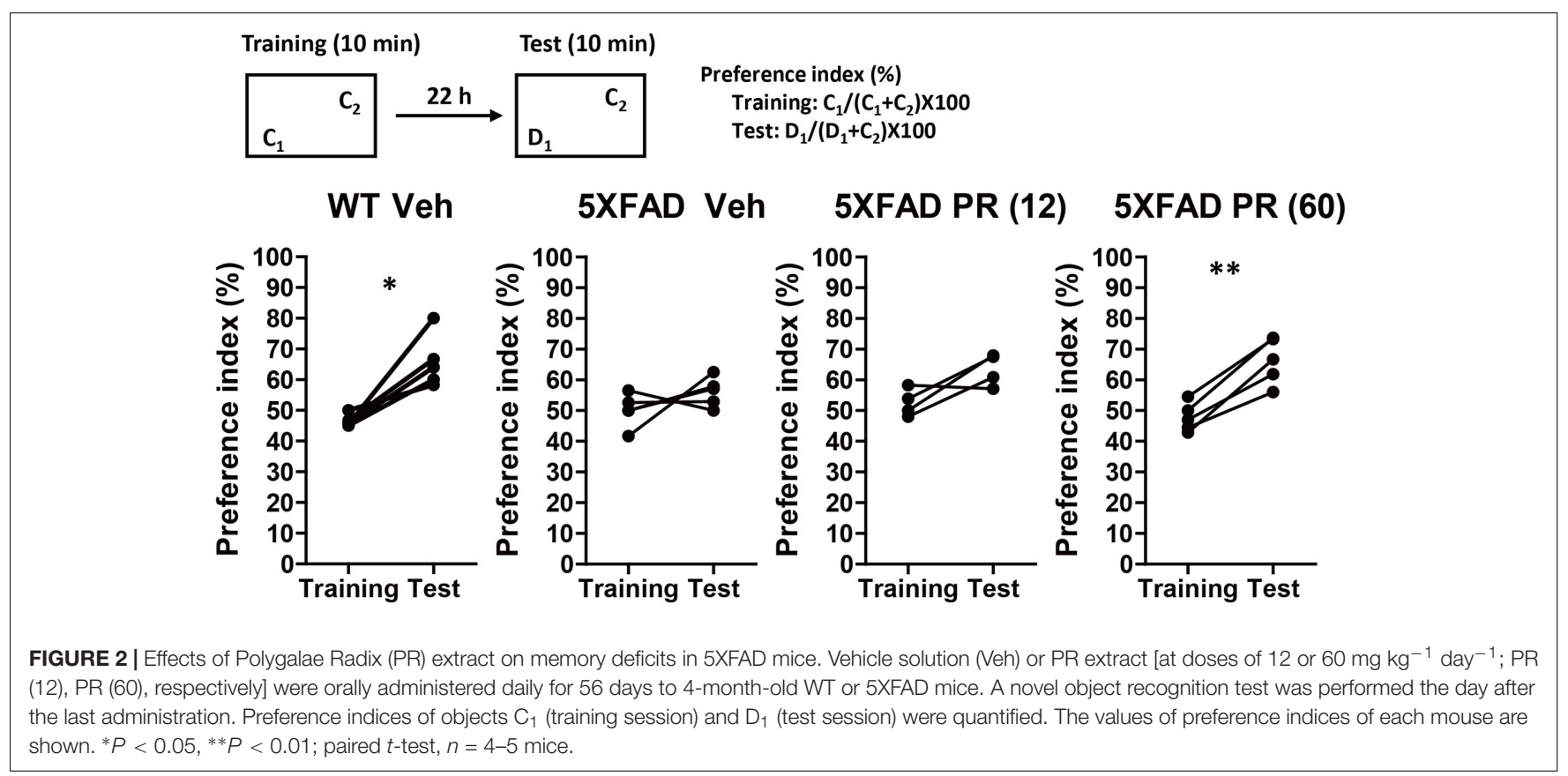

Endocytosis is crucial for $A \beta$-induced axonal collapse and axonal atrophy to occur in cultured neurons (Kuboyama et al., 2015). Additionally, inhibiting $A \beta$-induced endocytosis is associated with the amelioration of $\mathrm{A} \beta$-induced axonal degeneration and memory deficits in vivo (Kuboyama et al., 2015). Therefore, we investigated if PR extract inhibited $\mathrm{A} \beta$-induced endocytosis. To visualize endocytosis, cells were treated with a fluorescent dye, FM1-43FX, which binds to the surface of the cell plasma membrane, and together with the plasma membrane, is endocytosed into the cells. Only the endocytosed FM1-43FX can be visualized following twice wash. The neurons were fixed $20 \mathrm{~min}$ after treatment with A $\beta 1-42$, because FM1-43FX was endocytosed into the growth cones, but no axonal collapse was observed at this timepoint (Kuboyama et al., 2015). As shown in Figure 6, A $\beta 1-42$ increased endocytosis of FM1-43FX at the axonal endings. Pretreatment with PR extract significantly decreased the density of FM1-43FX-positive staining at axonal endings, indicating that the PR extract prevented endocytosis that was induced by $\mathrm{A} \beta 1-42$.

\section{DISCUSSION}

This study was the first to report that the PR extract can prevent cognitive dysfunction when consecutively administered orally to transgenic mice with $\mathrm{AD}$, at an age before the onset of memory deficits. Additionally, the PR extract also inhibited $\mathrm{A} \beta$-induced endocytosis and axon degeneration. Research previously conducted by our group demonstrated that 


\section{A}
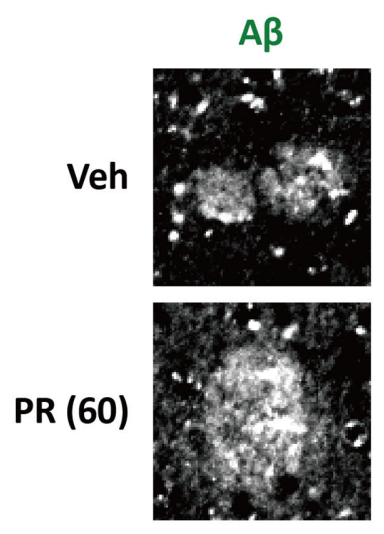

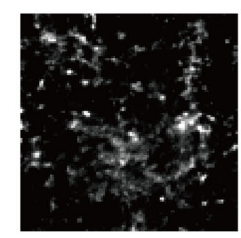

\section{Merged}
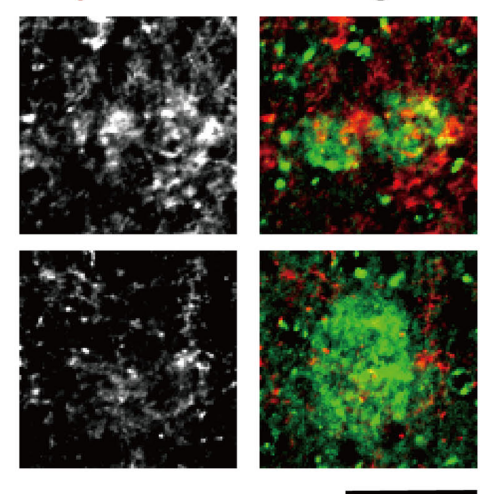

B

Perirhinal cortex

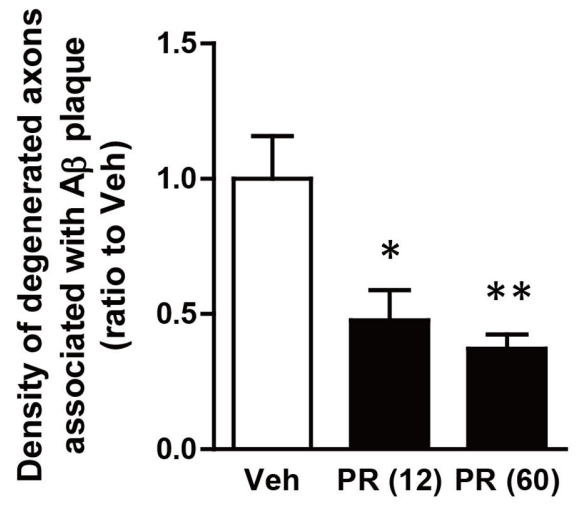

D

Perirhinal cortex

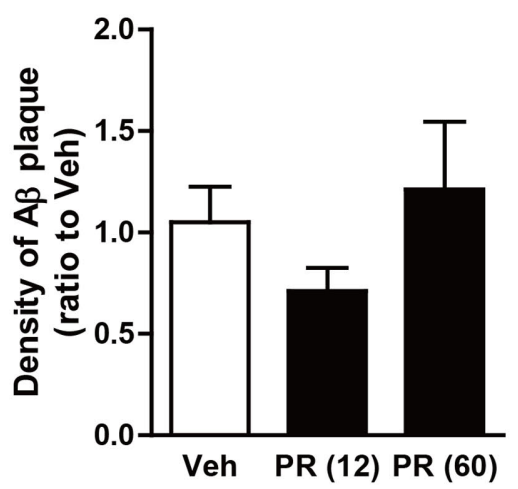

C

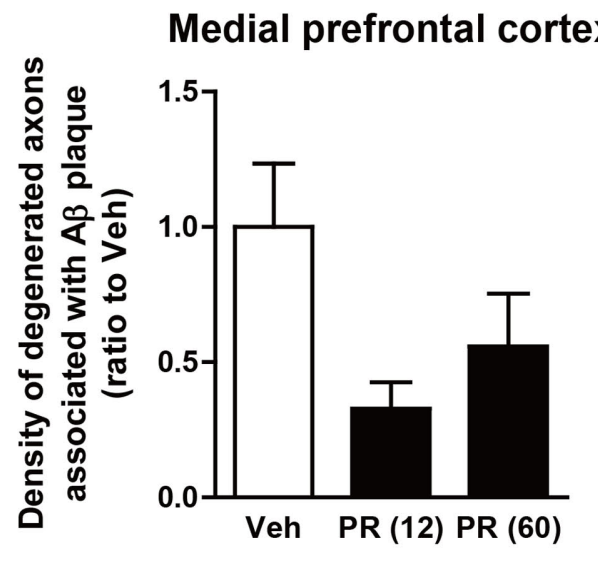

E

Medial prefrontal cortex

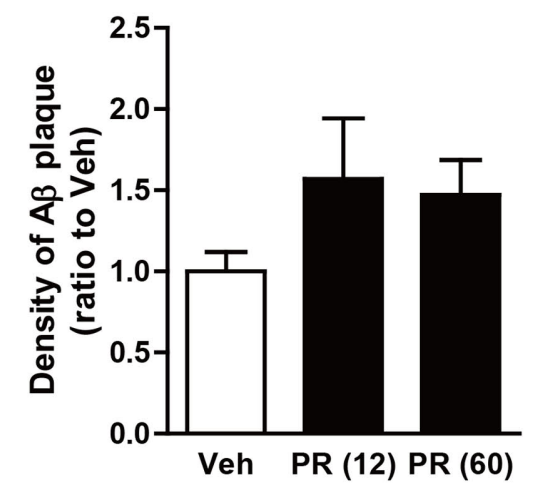

FIGURE 3 | Effects of Polygalae Radix (PR) extract on amyloid $\beta(A \beta)$ plaques and degenerated axons in 5XFAD mice. Vehicle solution (Veh) or PR extract [at doses of 12 or $60 \mathrm{mg} \mathrm{kg}^{-1}$ day $^{-1}$, PR (12), PR (60), respectively] were orally administered daily for 56 days to 4-month-old wild-type (WT) or 5XFAD mice. The mice were euthanized 2 days after the last administration, and the brains were sectioned and immunostained for phosphorylated neurofilament- $\mathrm{H}$ (pNF-H, an axonal marker, red) and $A \beta$ (green). (A) Representative fluorescence images of the perirhinal cortex are shown. Scale bar, $10 \mu \mathrm{m}$. (B,C) The density of degenerated axons associated with the $A \beta$ plaques was quantified in the perirhinal cortex $\mathbf{( B )}$ and medial prefrontal cortex (C). (D,E) The density of $A \beta$ plaques was quantified in the perirhinal cortex (D) and medial prefrontal cortex (E). The mean values of the data are presented together with the standard error. ${ }^{*} P<0.05,{ }^{* *} P<0.01$ vs. Veh; Bonferroni's multiple comparison test, $n=4-5$ mice. 


\section{Cont}

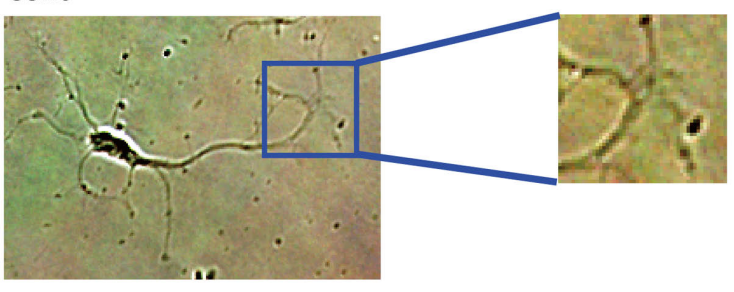

Veh/Aß1-42

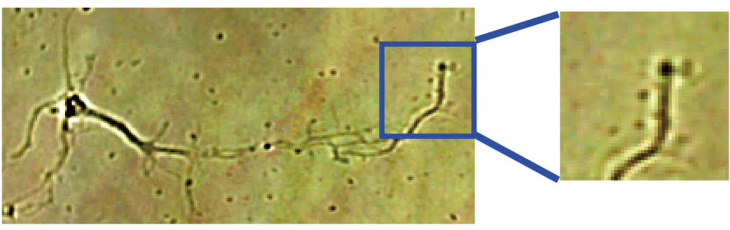

PR (100)/Aß1-42

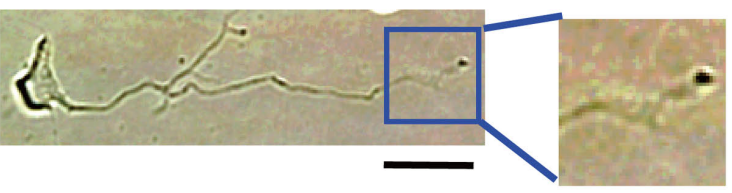

A 1 1-42

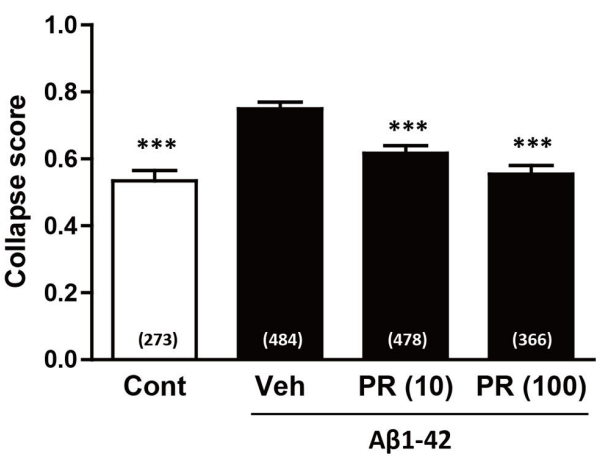

A 25-35

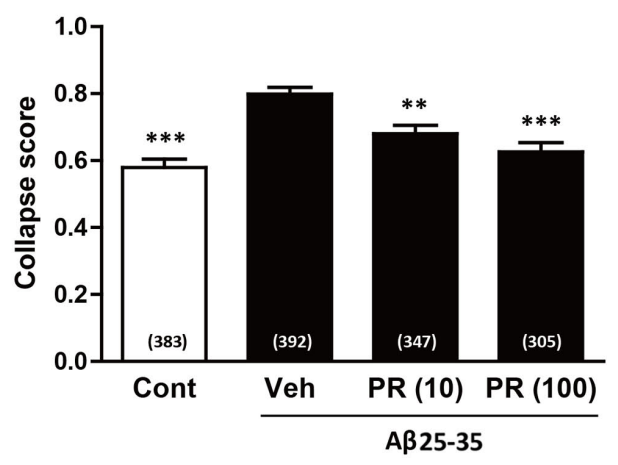

FIGURE 4 | Effects of the Polygalae Radix (PR) extract on amyloid $\beta$ (A $\beta$ )-induced growth cone collapse. Cortical neurons were cultured for 3 days, and treated for 30 min with vehicle solution (Cont, Veh) or PR extract [at doses of 10 or $100 \mu \mathrm{g} \mathrm{ml}^{-1}$; PR (10), PR (100), respectively]. Next, they were treated with vehicle solution (Cont), A $\beta 1-42(1 \mu \mathrm{M})$, or A $\beta 25-35(10 \mu \mathrm{M})$ for $1 \mathrm{~h}$. The neurons were then fixed. Representative bright field images with oblique illumination are shown (upper). Black scale bar, $20 \mu \mathrm{m}$. Blue scale bar, $10 \mu \mathrm{m}$. The growth cone shapes were quantified as collapse scores. The mean values of the data are presented together with the standard error (lower). The numbers in parentheses indicate the measured numbers of growth cones. ${ }^{* * P}<0.01, * * * P<0.001$ vs. Veh/A $\beta$; Dunn's multiple comparison test.

the intracerebroventricular administration of an endocytosis inhibitor, i.e., myristoylated dynamin inhibitory peptide or pitstop 2, prevented $A \beta$-induced memory deficits (Kuboyama et al., 2015). The effect of the systematic administration of those inhibitors has yet been investigated. Moreover, since many groups are trying to prevent or decrease $A \beta$ accumulation to prevent the progression of AD (Graham et al., 2017), no other group has ever reported a preventive drug for $\mathrm{AD}$ that works by inhibiting endocytosis. In contrast, our study shows that the oral administration of PR extract prevented memory deficits without decreasing $\mathrm{A} \beta$ deposition in 5XFAD mice. In this study, PR extract was identified as a potent endocytosis inhibitor, which prevented cognitive dysfunction without decreasing $\mathrm{A} \beta$ deposits in the mouse model for AD.

$\mathrm{A} \beta$ is believed to be a crucial cause for $\mathrm{AD}$ (Selkoe and Hardy, 2016). In humans, the level of $A \beta$ in the brain are upregulated before the onset of memory impairment (Jack and Holtzman, 2013). The accumulation of $A \beta$ in the brain is age-dependent, and neurodegeneration is induced before the appearance of memory function impairments. Thus, it can take a few decades for memory impairment to occur in patients with $\mathrm{AD}$. In 5XFAD mice, $\mathrm{A} \beta$ plaques and degenerated axons were observed at the age of 2 months and increased in an age-dependent manner (Supplementary Figure S2). In contrast, 


\section{PNF-H/MAP2/DAPI}

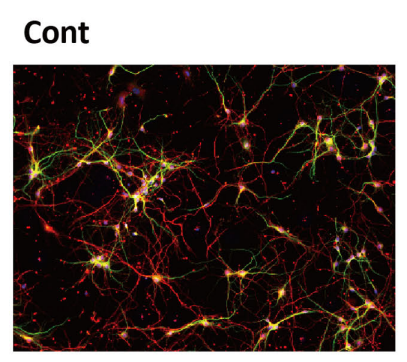

$A \beta /$ Veh

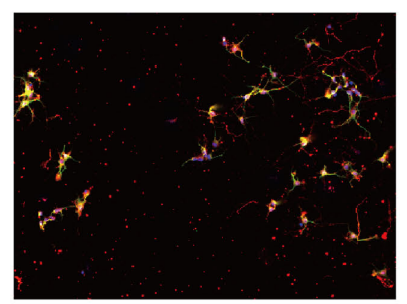

$A \beta / P R(10)$

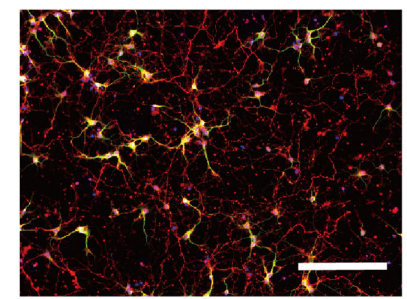

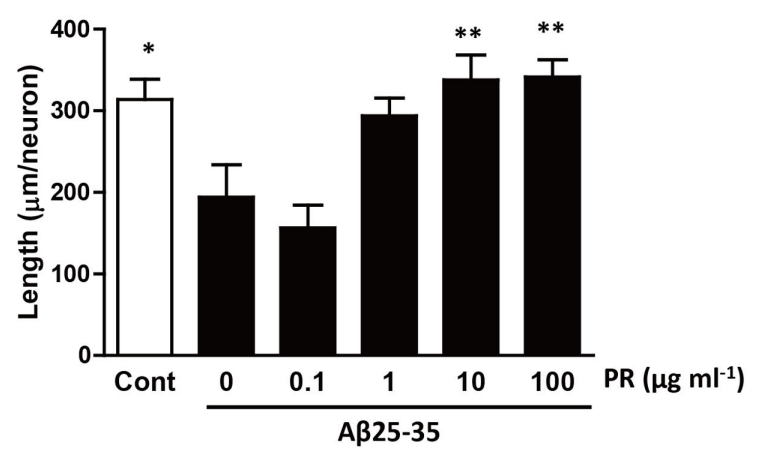

FIGURE 5 | Effects of Polygalae Radix (PR) extract on amyloid $\beta$ (A $\beta$ )-induced axonal atrophy. Cortical neurons were cultured for 3 days and treated simultaneously with vehicle solution (Cont) or A $25-35(10 \mu \mathrm{M})$, and vehicle solution (Cont, $0 \mu \mathrm{g} \mathrm{ml}^{-1}$ ) or PR extract (at doses of 0.1, 1, 10, or 100 $\mu \mathrm{g} \mathrm{ml}{ }^{-1}$ ) for $4 \mathrm{days}$. Thereafter, the neurons were fixed and immunostained for phosphorylated neurofilament-H (pNF-H, an axonal marker, red) and microtubule associated protein 2 (MAP2, a neuronal marker, green). DAPI (blue) was used for counterstaining. Representative fluorescence images are shown (upper). Scale bar, $200 \mathrm{\mu m}$. The total axonal length per neuron were quantified. The mean values of the data are presented together with the standard error (lower). $* * P<0.01 \mathrm{vs}$. $0 \mu \mathrm{g} / \mathrm{mL} \mathrm{PR}+\mathrm{A} \beta 25-35$; Bonferroni's multiple comparison test, $n=10$ images.

memory impairments only first occurred when the 5XFAD mice were 5 months old (Supplementary Figure S2). Memory impairment, in both humans and mice, seems to be evident when $\mathrm{A} \beta$ and/or neurodegeneration levels exceed a certain threshold. In the current study, the PR extract inhibited axonal degeneration without decreasing $\mathrm{A} \beta$ deposition, thus preserving memory function in the 5XFAD mice. Although many clinical studies have focused on clearing $A \beta$ from the brain, none have succeeded in preserving or recovering memory function in patients with AD (Graham et al., 2017). Since A $\beta$ abundantly accumulates in the brain before the $\mathrm{AD}$ symptoms appear (Selkoe and Hardy, 2016), treatment that focuses on clearing $A \beta$ may be belated and insufficient to repair $A \beta$-induced disruption of neural networks. Therefore, rather than focusing on $A \beta$, the downstream signaling of $A \beta$ could be a better target for preventing axon degeneration in $\mathrm{AD}$.

A $\beta$-induced endocytosis can lead to axonal collapse, preventing axonal growth, and consequently causing axonal degeneration (Kuboyama et al., 2015). Endocytosis is thought to reduce the surface area of the plasma membrane and eliminate some essential molecules from the surface of the growth cone (Tojima et al., 2011). After endocytosis of the plasma membrane, the growth cone is unable to maintain its own morphology, and axonal collapse occurs. $\mathrm{A} \beta$ also reportedly induced synaptic dysfunction, via endocytosis of $\alpha$-amino-3-hydroxy-5-methyl4 -isoxazolepropionic acid receptors and $N$-methyl-D-aspartic acid receptors (Snyder et al., 2005; Kurup et al., 2010; Zhao et al., 2010). Amyloid precursor protein on the plasma membrane is endocytosed and cleaved to produce $A \beta$, which is then released into the interstitial fluid of the brain (Cirrito et al., 2008). Approximately $70 \%$ of $A \beta$ in the interstitial fluid was predicted to be produced via the endocytosis pathway (Cirrito et al., 2008). Therefore, the inhibition of endocytosis is certainly a promising target for the prevention of $\mathrm{AD}$.

Tenuifolin, a component of $\mathrm{PR}$, was reported to decrease the secretion of $A \beta$ from COS-7 cells expressing the mutant form of amyloid precursor protein (Lv et al., 2009). Tenuifolin also counteracts $\mathrm{A} \beta 25-35$-induced neurotoxicity in vitro and in vivo, improving spatial memory (Liu et al., 2015). A hydrolysate of a polygalasaponin fraction derived from $\mathrm{PR}$ enhanced spatial memory in A $\beta 25$-35-induced amnesic mice, probably via its antioxidative activity (Xu et al., 2011). Antioxidants are expected to prevent AD (Vina et al., 2004; Ray et al., 2011a,b). Therefore, these pharmacological effects also possibly contributed to the preventive effects of $\mathrm{PR}$ extract in $\mathrm{AD}$. However, these studies were performed only in cultured cells or $A \beta$-injected animal models. In $\mathrm{AD}, \mathrm{A} \beta$ deposition and cognitive dysfunction occur in an age-dependent manner. Thus, to examine the preventive activities of drugs against $\mathrm{AD}$ in a preclinical study, transgenic animal models of $\mathrm{AD}$, which show $\mathrm{AD}$ phenotypes in an agedependent manner, should be used. To our knowledge, the current study is the first to show the preventive effects of 


\section{Cont}
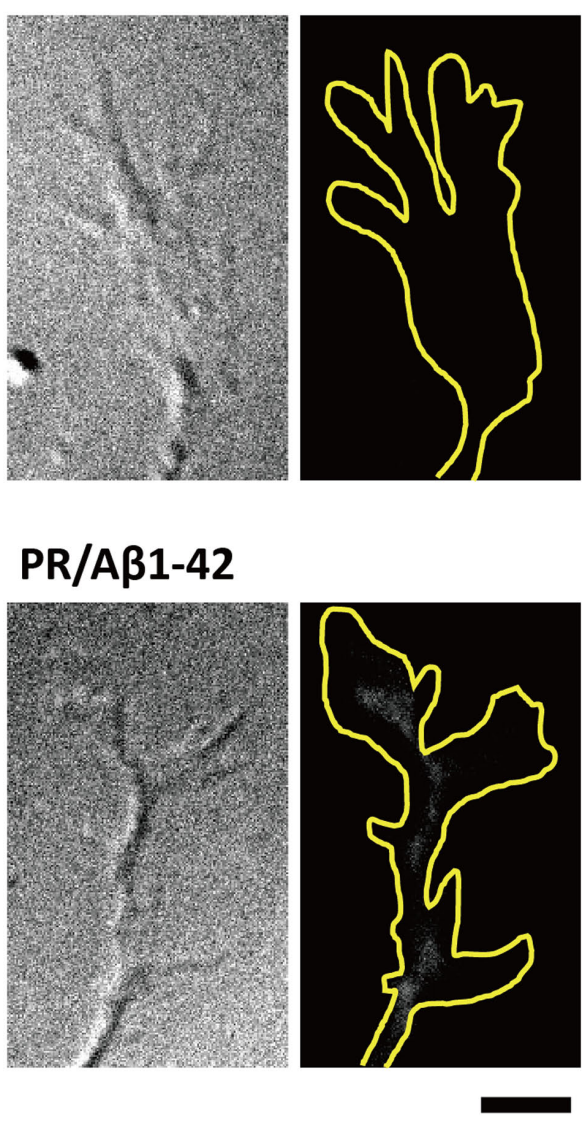

\section{Veh/Aß1-42}
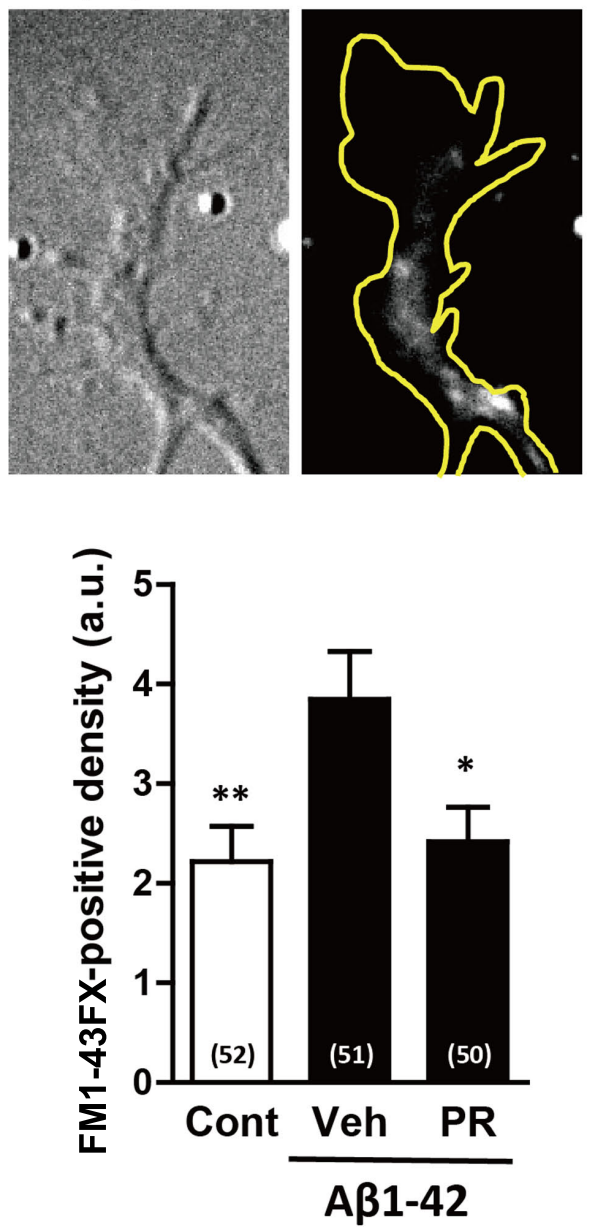

FIGURE 6 | Effects of Polygalae Radix (PR) extract on amyloid $\beta(A \beta)$-induced endocytosis. Cortical neurons were cultured for 3 days, and treated with vehicle solution (Cont) or A $\beta 1-42(1 \mu \mathrm{M})$ for $30 \mathrm{~min}$. Next, they were treated with FM1-43FX (20 $\mu \mathrm{M})$ for 1 min, and vehicle solution (Cont, Veh) or PR extract (100 $\mu \mathrm{g} \mathrm{ml}^{-1}$ ) for $20 \mathrm{~min}$, before the neurons were fixed. Representative differential interference contrast and fluorescence images are shown. The yellow dotted lines represent outlines of the growth cone. Scale bar, $5 \mu \mathrm{m}$. The density of FM1-43FX-positive area in each growth cone were quantified. The mean values of the data are presented together with the standard error. The numbers in parentheses indicate the measured numbers of growth cones. ${ }^{*} P<0.05$, ${ }^{*} P<0.01$ vs. Veh/A $\beta 1-42$; Bonferroni's multiple comparison test.

$\mathrm{PR}$ against the $\mathrm{AD}$ phenotype in a transgenic mouse model of $\mathrm{AD}$.

BT-11, a crude extract of PR, was reported to improve spatial memory in rats suffering from chronic stress or treated with scopolamine (Park et al., 2002; Shin et al., 2009). BT-11 also reportedly enhanced memory function in healthy humans (Lee et al., 2009). PR has traditionally been used in a clinical capacity for more than a thousand years in Japan, China, and South Korea. Therefore, the safety of PR for clinical use has already been established. In our study, the chronic administration of PR extract for 2 months did not influence body weight or general behaviors. Considering that PR has been traditionally been used for memory enhancement, PR extract could be used as a safe preventive drug for $\mathrm{AD}$.

Next, we assessed if the administration of PR extract after the onset of $\mathrm{AD}$ pathologies could ameliorate cognitive dysfunction in $\mathrm{AD}$. We previously demonstrated that $\mathrm{PR}$ extract promoted axon elongation in cultured neurons after $A \beta$ had already induced axonal degeneration (Naito and Tohda, 2006). Several herbal drugs, which induced axon elongation in neurons damaged by $A \beta$, also showed the recovery of memory function in aged 5XFAD mice showing memory impairments (Joyashiki et al., 2011; Tohda et al., 2011, 2012; Yang et al., 2017). Tenuifolin and polygalasaponin hydrate derived from PR also led to recovery of memory after A $\beta 25-$ 35 was injected into the hippocampus of the mice (Xu et al., 2011; Liu et al., 2015). These results indicated that PR extract could putatively enhance the recovery of memory in patients with $\mathrm{AD}$.

In the current study, we clarified the preventive effects of PR extract on axon degeneration and memory impairment in 5XFAD transgenic AD mice, and found a novel pharmacological 
activity of the PR extract, i.e., the inhibition of endocytosis. As described above, PR extract and its constituents show various pharmacological effects possibly preventing $\mathrm{AD}$. PR extract could be the first multi-functional preventive drug against $\mathrm{AD}$, at least partly due to its ability to inhibit endocytosis.

\section{AUTHOR CONTRIBUTIONS}

TK and CT designed the experiments and wrote the manuscript. $\mathrm{KH}, \mathrm{TA}$, and $\mathrm{HY}$ performed and analyzed HPLC experiments. TK performed and analyzed all other experiments.

\section{FUNDING}

This work was partially supported by a fund from Kobayashi Pharmaceutical Co., Ltd., Japan, the Discretionary Fund of the President and 2017 Director Leadership Expenses in the University of Toyama, Japan, and Takeda Science Foundation, Japan.

\section{ACKNOWLEDGMENT}

We would like to thank Editage (www.editage.jp) for English language editing.

\section{REFERENCES}

Barker, G. R., and Warburton, E. C. (2011). When is the hippocampus involved in recognition memory? J. Neurosci. 31, 10721-10731. doi: 10.1523/jneurosci. 6413-10.2011

Benes, F. M., Farol, P. A., Majocha, R. E., Marotta, C. A., and Bird, E. D. (1991). Evidence for axonal loss in regions occupied by senile plaques in Alzheimer cortex. Neuroscience 42, 651-660. doi: 10.1016/0306-4522(91) 90034-L

Chinese Pharmacopoeia Commission (2015). "Polygalae radix," in Pharmacopoeia of the People's Republic of China, eds Y. Zhao, Z. Fan, K. Huang, Z. Li, and Y. Gao (Beijing: People's Medicine Publishing House Press).

Cirrito, J. R., Kang, J. E., Lee, J., Stewart, F. R., Verges, D. K., Silverio, L. M., et al. (2008). Endocytosis is required for synaptic activity-dependent release of amyloid-beta in vivo. Neuron 58, 42-51. doi: 10.1016/j.neuron.2008. 02.003

Dickson, T. C., and Vickers, J. C. (2001). The morphological phenotype of beta-amyloid plaques and associated neuritic changes in Alzheimer's disease. Neuroscience 105, 99-107. doi: 10.1016/S0306-4522(01)00169-5

Dotti, C. G., Sullivan, C. A., and Banker, G. A. (1988). The establishment of polarity by hippocampal neurons in culture. J. Neurosci. 8, 1454-1468.

Faul, F., Erdfelder, E., Lang, A. G., and Buchner, A. (2007). G*Power 3: a flexible statistical power analysis program for the social, behavioral, and biomedical sciences. Behav. Res. Methods 39, 175-191. doi: 10.3758/BF03193146

Graham, W. V., Bonito-Oliva, A., and Sakmar, T. P. (2017). Update on Alzheimer's disease therapy and prevention strategies. Annu. Rev. Med. 68, 413-430. doi: 10.1146/annurev-med-042915-103753

Hardy, J., and Selkoe, D. J. (2002). The amyloid hypothesis of Alzheimer's disease: progress and problems on the road to therapeutics. Science 297, 353-356. doi: $10.1126 /$ science. 1072994

Jack, C. R. Jr., and Holtzman, D. M. (2013). Biomarker modeling of Alzheimer's disease. Neuron 80, 1347-1358. doi: 10.1016/j.neuron.2013.12.003

\section{SUPPLEMENTARY MATERIAL}

The Supplementary Material for this article can be found online at: https://www.frontiersin.org/articles/10.3389/fphar. 2017.00805/full\#supplementary-material

FIGURE S1 | High performance liquid chromatography (HPLC) profiles. Polygalae Radix (PR) extract and standard compounds were analyzed using two kinds of HPLC protocols, i.e., one to detect tenuifolin $(\mathbf{A}, \mathbf{B})$ and the other to detect of $3^{\prime}, 6$-di-O-sinapoyl sucrose ester (C,D). HPLC profiles of PR extract $(\mathbf{A}, \mathbf{C})$ and standard compounds, tenuifolin (B) and $3^{\prime}, 6$-di-O-sinapoyl sucrose ester (D), are shown. Arrows indicate peaks of tenuifolin (A,B) and 3',6-di-O-sinapoyl sucrose ester $\mathbf{( C , D )}$, respectively.

FIGURE S2 | Age-dependent phenotypes of 5XFAD mice. (A) Novel object recognition tests were performed as done in Figure 2 using WT and 5XFAD male mice at various ages. The numbers in parentheses indicate the age of the mice. Values of preferential indices of each mouse are shown. Paired $t$-test; $n=3$ mice. (B,C) After the behavioral tests, the mice were euthanized and the brain sections were immunostained for phosphorylated neurofilament- $\mathrm{H}(\mathrm{pNF}-\mathrm{H})$ and amyloid $\beta$ $(A \beta)$. The density of $A \beta$ plaques $(\mathbf{B})$ and degenerated axons associated with the $A \beta$ plaque $\mathbf{( C )}$ were quantified in the medial prefrontal cortex. The mean values of the data are presented together with the standard error. ${ }^{* * *} P<0.001$ vs. WT; Bonferroni's multiple comparison test. $n=3$ mice.

FIGURE S3 | Changes in body weight and exploratory behaviors after the administration of PR extract. Vehicle solution (Veh) or PR extract [at doses of 12 or 60 mg/kg/day; PR (12), PR (60), respectively] were orally administered daily for 56 days to 4-month-old 5XFAD mice. (A) The mean values of the body weight of the mice are presented together with the standard error. There is no statistical difference among groups. $P>0.05$; 2 -way repeated measures ANOVA, $n=4-5$ mice. (B) Total number of times the mice encountered the two objects in the open box was counted in the training session. There was no statistical difference among the groups. $P>0.05$; 1 -way ANOVA, $n=4-5$ mice.

Jack, C. R. Jr., Knopman, D. S., Jagust, W. J., Shaw, L. M., Aisen, P. S., Weiner, M. W., et al. (2010). Hypothetical model of dynamic biomarkers of the Alzheimer's pathological cascade. Lancet Neurol. 9, 119-128. doi: 10.1016/ s1474-4422(09)70299-6

Jia, H., Jiang, Y., Ruan, Y., Zhang, Y., Ma, X., Zhang, J., et al. (2004). Tenuigenin treatment decreases secretion of the Alzheimer's disease amyloid beta-protein in cultured cells. Neurosci. Lett. 367, 123-128. doi: 10.1016/j.neulet.2004. 05.093

Joyashiki, E., Matsuya, Y., and Tohda, C. (2011). Sominone improves memory impairments and increases axonal density in Alzheimer's disease model mice, 5XFAD. Int. J. Neurosci. 121, 181-190. doi: 10.3109/00207454.2010.541571

Jurney, W. M., Gallo, G., Letourneau, P. C., and McLoon, S. C. (2002). Rac1mediated endocytosis during ephrin-A2- and semaphorin 3A-induced growth cone collapse. J. Neurosci. 22, 6019-6028.

Killin, L. O., Starr, J. M., Shiue, I. J., and Russ, T. C. (2016). Environmental risk factors for dementia: a systematic review. BMC Geriatr. 16:175. doi: 10.1186/ s12877-016-0342-y

Kuboyama, T., Lee, Y. A., Nishiko, H., and Tohda, C. (2015). Inhibition of clathrin-mediated endocytosis prevents amyloid beta-induced axonal damage. Neurobiol. Aging 36, 1808-1819. doi: 10.1016/j.neurobiolaging.2015.02.005

Kuboyama, T., Tohda, C., and Komatsu, K. (2005). Neuritic regeneration and synaptic reconstruction induced by withanolide A. Br. J. Pharmacol. 144, 961-971. doi: 10.1038/sj.bjp.0706122

Kurup, P., Zhang, Y., Xu, J., Venkitaramani, D. V., Haroutunian, V., Greengard, P., et al. (2010). A $\beta$-Mediated NMDA receptor endocytosis in Alzheimer's Disease Involves Ubiquitination of the Tyrosine Phosphatase STEP. J. Neurosci. 30, 5948-5957. doi: 10.1523/jneurosci.0157-10.2010

Lee, J. Y., Kim, K. Y., Shin, K. Y., Won, B. Y., Jung, H. Y., and Suh, Y. H. (2009). Effects of BT-11 on memory in healthy humans. Neurosci. Lett. 454, 111-114. doi: 10.1016/j.neulet.2009.03.024

Liu, Y. M., Li, Z. Y., Hu, H., Xu, S. P., Chang, Q., Liao, Y. H., et al. (2015). Tenuifolin, a secondary saponin from hydrolysates of polygalasaponins, 
counteracts the neurotoxicity induced by Abeta25-35 peptides in vitro and in vivo. Pharmacol. Biochem. Behav. 128, 14-22. doi: 10.1016/j.pbb.2014. 11.010

Lv, J., Jia, H., Jiang, Y., Ruan, Y., Liu, Z., Yue, W., et al. (2009). Tenuifolin, an extract derived from tenuigenin, inhibits amyloid-beta secretion in vitro. Acta Physiol. (196, 419-425. doi: 10.1111/j.1748-1716.2009.01961.x

Maloney, B., and Lahiri, D. K. (2016). Epigenetics of dementia: understanding the disease as a transformation rather than a state. Lancet Neurol. 15, 760-774. doi: 10.1016/s1474-4422(16)00065-x

Masliah, E., Mallory, M., Hansen, L., Alford, M., DeTeresa, R., and Terry, R. (1993). An antibody against phosphorylated neurofilaments identifies a subset of damaged association axons in Alzheimer's disease. Am. J. Pathol. 142, 871-882.

May, B. H., Lu, C., Lu, Y., Zhang, A. L., and Xue, C. C. (2013). Chinese herbs for memory disorders: a review and systematic analysis of classical herbal literature. J. Acupunct. Meridian Stud. 6, 2-11. doi: 10.1016/j.jams.2012.11.009

Naito, R., and Tohda, C. (2006). Characterization of anti-neurodegenerative effects of Polygala tenuifolia in Abeta(25-35)-treated cortical neurons. Biol. Pharm. Bull. 29, 1892-1896. doi: 10.1248/bpb.29.1892

Oakley, H., Cole, S. L., Logan, S., Maus, E., Shao, P., Craft, J., et al. (2006). Intraneuronal beta-amyloid aggregates, neurodegeneration, and neuron loss in transgenic mice with five familial Alzheimer's disease mutations: potential factors in amyloid plaque formation. J. Neurosci. 26, 10129-10140. doi: 10.1523/ JNEUROSCI.1202-06.2006

Park, C. H., Choi, S. H., Koo, J. W., Seo, J. H., Kim, H. S., Jeong, S. J., et al. (2002). Novel cognitive improving and neuroprotective activities of Polygala tenuifolia Willdenow extract, BT-11. J. Neurosci. Res. 70, 484-492. doi: 10.1002/jnr. 10429

Perl, D. P. (2010). Neuropathology of Alzheimer's disease. Mt. Sinai J. Med. 77, 32-42. doi: $10.1002 / \mathrm{msj} .20157$

Pimenova, A. A., Raj, T., and Goate, A. M. (2017). Untangling genetic risk for Alzheimer's disease. Biol. Psychiatry doi: 10.1016/j.biopsych.2017.05.014 [Epub ahead of print].

Ray, B., Bisht, S., Maitra, A., Maitra, A., and Lahiri, D. K. (2011a). Neuroprotective and neurorescue effects of a novel polymeric nanoparticle formulation of curcumin (NanoCurc) in the neuronal cell culture and animal model: implications for Alzheimer's disease. J. Alzheimers. Dis. 23, 61-77. doi: 10.3233/ jad-2010-101374

Ray, B., Chauhan, N. B., and Lahiri, D. K. (2011b). Oxidative insults to neurons and synapse are prevented by aged garlic extract and S-allyl-1-cysteine treatment in the neuronal culture and APP-Tg mouse model. J. Neurochem. 117, 388-402. doi: $10.1111 /$ j.1471-4159.2010.07145.x

Selkoe, D. J., and Hardy, J. (2016). The amyloid hypothesis of Alzheimer's disease at 25 years. EMBO Mol. Med. 8, 595-608. doi: 10.15252/emmm. 201606210

Shin, K. Y., Won, B. Y., Heo, C., Kim, H. J., Jang, D. P., Park, C. H., et al. (2009). BT-11 improves stress-induced memory impairments through increment of glucose utilization and total neural cell adhesion molecule levels in rat brains. J. Neurosci. Res. 87, 260-268. doi: 10.1002/jnr.21834

Simmons, D. A., Knowles, J. K., Belichenko, N. P., Banerjee, G., Finkle, C., Massa, S. M., et al. (2014). A small molecule p75NTR ligand, LM11A-31, reverses cholinergic neurite dystrophy in Alzheimer's disease mouse models with midto late-stage disease progression. PLOS ONE 9:e102136. doi: 10.1371/journal. pone. 0102136

Snyder, E. M., Nong, Y., Almeida, C. G., Paul, S., Moran, T., Choi, E. Y., et al. (2005). Regulation of NMDA receptor trafficking by amyloid-beta. Nat. Neurosci. 8, 1051-1058. doi: 10.1038/nn1503
Tanimizu, T., Kono, K., and Kida, S. (2017). Brain networks activated to form object recognition memory. Brain Res. Bull. doi: 10.1016/j.brainresbull.2017.05.017 [Epub ahead of print].

Tohda, C. (2016). New age therapy for Alzheimer's disease by neuronal network reconstruction. Biol. Pharm. Bull. 39, 1569-1575. doi: 10.1248/bpb.b16-00438

Tohda, C., Nakada, R., Urano, T., Okonogi, A., and Kuboyama, T. (2011). Kamikihi-to (KKT) rescues axonal and synaptic degeneration associated with memory impairment in a mouse model of Alzheimer's disease, 5XFAD. Int. J. Neurosci. 121, 641-648. doi: 10.3109/00207454.2011.602809

Tohda, C., Urano, T., Umezaki, M., Nemere, I., and Kuboyama, T. (2012). Diosgenin is an exogenous activator of 1,25D3-MARRS/Pdia3/ERp57 and improves Alzheimer's disease pathologies in 5XFAD mice. Sci. Rep. 2:535. doi: 10.1038/srep00535

Tojima, T., Hines, J. H., Henley, J. R., and Kamiguchi, H. (2011). Second messengers and membrane trafficking direct and organize growth cone steering. Nat. Rev. Neurosci. 12, 191-203. doi: 10.1038/nrn2996

Urano, T., and Tohda, C. (2010). Icariin improves memory impairment in Alzheimer's disease model mice $(5 x F A D)$ and attenuates amyloid beta-induced neurite atrophy. Phytother. Res. 24, 1658-1663. doi: 10.1002/ptr.3183

Vilberg, K. L., and Davachi, L. (2013). Perirhinal-hippocampal connectivity during reactivation is a marker for object-based memory consolidation. Neuron 79 , 1232-1242. doi: 10.1016/j.neuron.2013.07.013

Vina, J., Lloret, A., Orti, R., and Alonso, D. (2004). Molecular bases of the treatment of Alzheimer's disease with antioxidants: prevention of oxidative stress. Mol. Aspects Med. 25, 117-123. doi: 10.1016/j.mam.2004.02.013

Wu, T. Y., Chen, C. P., and Jinn, T. R. (2011). Traditional Chinese medicines and Alzheimer's disease. Taiwan. J. Obstet. Gynecol. 50, 131-135. doi: 10.1016/j.tjog. 2011.04.004

Xu, S. P., Yang, Y. Y., Xue, D., Liu, J. X., Liu, X. M., Fan, T. P., et al. (2011). Cognitive-enhancing effects of polygalasaponin hydrolysate in abeta(25-35)-induced amnesic mice. Evid. Based Complement. Alternat. Med. 2011:839720. doi: 10.1155/2011/839720

Yang, Z., Kuboyama, T., and Tohda, C. (2017). A systematic strategy for discovering a therapeutic drug for Alzheimer's disease and its target molecule. Front. Pharmacol. 8:340. doi: 10.3389/fphar.2017.00340

Yankner, B. A., Duffy, L. K., and Kirschner, D. A. (1990). Neurotrophic and neurotoxic effects of amyloid beta protein: reversal by tachykinin neuropeptides. Science 250, 279-282. doi: 10.1126/science.2218531

Zhao, W. Q., Santini, F., Breese, R., Ross, D., Zhang, X. D., Stone, D. J., et al. (2010). Inhibition of calcineurin-mediated endocytosis and alpha-amino-3-hydroxy5-methyl-4-isoxazolepropionic acid (AMPA) receptors prevents amyloid beta oligomer-induced synaptic disruption. J. Biol. Chem. 285, 7619-7632. doi: 10.1074/jbc.M109.057182

Conflict of Interest Statement: The authors would like to disclose that they received research funding from Kobayashi Pharmaceutical Co., Ltd. to conduct this study. The authors declare that the funders had no role in the study design, data collection and analysis, decision to publish or preparation of the manuscript.

Copyright (c) 2017 Kuboyama, Hirotsu, Arai, Yamasaki and Tohda. This is an openaccess article distributed under the terms of the Creative Commons Attribution License (CC BY). The use, distribution or reproduction in other forums is permitted, provided the original author(s) or licensor are credited and that the original publication in this journal is cited, in accordance with accepted academic practice. No use, distribution or reproduction is permitted which does not comply with these terms. 